\title{
0 que funciona e 0 que não funciona para reduzir homicídios no Brasil: uma revisão sistemática
}

\author{
Alberto L. W. Kopittke 12 \\ Marília Patta Ramos ${ }^{3}$ \\ ${ }^{1}$ Universidade Federal do Rio Grande do Sul, Porto Alegre / RS - Brasil \\ 2 Instituto Cidade Segura, Porto Alegre / RS - Brasil \\ 3 Universidade Federal do Rio Grande do Sul / Departamento de Sociologia, Porto Alegre / RS - Brasil
}

Este artigo tem por objetivo identificar quais são as evidências científicas existentes no Brasil, hoje, sobre o que funciona e o que não funciona para reduzir o número de homicídios, por meio da primeira revisão sistemática já realizada no país acerca da efetividade de programas de segurança pública, utilizando a mesma metodologia do Relatório Maryland, realizado em 1997, o qual revisou os estudos já produzidos em língua inglesa e que é considerado um marco no surgimento da chamada segurança pública baseada em evidências (SPBE). A busca resultou em um total de 13.352 estudos analisados, dos quais 41 preencheram os critérios de inclusão, resultando na identificação de 8 tipos de programas que funcionam, 7 programas promissores, 7 programas com resultados indefinidos e 2 programas com evidências que não funcionam. O estudo mostrou que o país dispõe de programas inovadores que são efetivos para reduzir homicídios, enquanto ações tradicionalmente citadas como soluções para a área não apresentam evidências científicas de que funcionem. Embora atrasado em relação ao desenvolvimento internacional, o paradigma da SPBE apresenta grande potencial para aumentar a efetividade do gasto público, auxiliar o país a reduzir a violência e superar processos de tomada de decisão baseados em crenças, corporativismos, preconceitos e populismos políticos.

Palavras-chave: segurança pública baseada em evidências; políticas públicas baseadas em evidências; prevenção à violência; polícia e democracia.

\section{Lo que funciona y lo que no funciona para reducir los homicidios en Brasil: una revisión sistemática}

Este artículo tiene como objetivo identificar qué evidencia científica existe hoy en Brasil sobre lo que funciona y lo que no funciona para reducir el número de homicidios, a través de la primera revisión sistemática que se ha realizado en el país sobre la eficacia de los programas de seguridad pública, utilizando la misma metodología del Informe Maryland, realizado en 1997, que revisó los estudios ya realizados en inglés y que se considera un hito en el surgimiento de la llamada seguridad pública basada en la evidencia (SPBE). La búsqueda resultó en un total de 13.352 estudios analizados, de los cuales 41 cumplieron los criterios de inclusión, lo que dio como resultado la identificación de 8 tipos de programas que funcionan, 7 programas prometedores, 7 programas con resultados no definidos y 2 programas con pruebas de que no funcionan. El estudio demostró que el país tiene programas innovadores que son eficaces para reducir los homicidios, mientras que las acciones tradicionalmente citadas como soluciones para el área no presentan evidencia científica de que funcionen. Aunque va a la zaga del desarrollo internacional, el paradigma de la SPBE presenta gran potencial para aumentar la eficacia del gasto público, ayudar al país a reducir la violencia y superar los procesos de toma de decisiones basados en creencias, corporativismo, prejuicios y populismo político.

Palabras clave: seguridad pública basada en la evidencia; políticas públicas basadas en la evidencia; prevención de la violencia; policía y democracia. 


\section{What works and what does not work to reduce homicides in Brazil: a systematic review}

This article aims to identify the scientific evidence in Brazil on what works and what does not work to reduce the number of homicides. This is the first systematic review ever conducted in the country on the effectiveness of public security programs, using the same methodology as the Maryland Report, conducted in 1997, which reviewed the studies produced in English and is considered a milestone in the emergence of the so-called evidence-based crime prevention paradigm (EBCP). The review resulted in a total of 13,352 studies, of which 41 met the inclusion criteria, resulting in the identification of 8 types of programs that work, 7 promising programs, 7 programs with undefined results, and 2 programs with evidence that shows they do not work. The study showed that the country has innovative programs that effectively reduce homicides, while actions traditionally cited as solutions do not present scientific evidence that they work. Although lagging behind international development, the EBCP presents the potential to increase the effectiveness of public spending, help the country to reduce violence, and overcome decision-making processes based on beliefs, corporatism, prejudice, and political populism.

Keywords: crime prevention evidence-based; evidence-based public policies; violence prevention; policing and democracy.

\section{AGRADECIMENTOS}

A pesquisa foi desenvolvida no âmbito do Programa de Pós-Graduação em Políticas Públicas da Universidade Federal do Rio Grande do Sul. Os autores agradecem o auxílio inestimável de Fernando Gonçalvez, Juliana Escobar e Liciane Barbosa que participaram do processo de realização da Revisão Sistemática.

\section{INTRODUÇÃO}

O Brasil sofre, há meio século, as consequências de uma epidemia de violência e da incapacidade do Poder Público para lidar, de modo eficiente, com esse grave problema. Entre 1980 e 2019, o país teve em média 40 mil homicídios por ano, resultando em pelo menos 1,5 milhão de brasileiros assassinados (Instituto de Pesquisa Econômica Aplicada \& Fórum Brasileiro de Segurança Pública, 2019) e a população prisional aumentou mais de 700\% (Santos \& Rosa, 2017). Apesar do país ter apenas 2,8\% da população mundial, 15,3\% de todos os assassinatos do mundo, em 2016, ocorreram em território brasileiro (Global Burden of Disease Collaborative Network [GBD], 2017) e o país tinha, em 2018, 17 cidades entre as 50 mais violentas do mundo (Seguridad, Justicia y Paz, 2019).

Em resposta a essa situação, os diferentes níveis de governo e poderes já realizaram diversas ações e gastaram um volume bastante expressivo de recursos. O Governo Federal, desde a redemocratização, já lançou pelo menos 4 Planos Nacionais de Segurança (Kopittke, 2016), realizou 101 operações de Garantia da Lei e da Ordem (GLO) por parte das Forças Armadas (Ministério da Defesa, 2018), centenas de operações da Polícia Federal (PF) e uma Intervenção Federal no Estado do Rio de Janeiro. Além disso, o Congresso Nacional aprovou uma média de 5 novas legislações federais sobre segurança pública e política criminal por ano (Campos, 2010). No entanto, até o momento, o gasto público na área é pouco eficiente e em geral muito pouco efetivo para reduzir os índices de violência.

Um dos motivos que têm sido apontados para o insucesso das ações de segurança pública no Brasil é o fato do país não conseguir avançar na produção e no uso de evidências científicas para resolver os problemas de violência, como tem ocorrido em nível internacional com o avanço da chamada 
Segurança Pública Baseada em Evidências (SPBE) (Beato, 2002; Beato \& Silveira, 2014; Cano, Rojido, Chaves, Bezerra \& Sá, 2016; Rolim, 2006; Willadino, Sento-Sé, Dias \& Gomes, 2011).

Os países com democracia consolidada também vivenciaram, entre os anos 1960 e 1980, uma profunda crise de efetividade, eficiência e legitimidade do chamado modelo tradicional de segurança pública, que se baseava, até então, exclusivamente em ações reativas, sem foco e sem integração das polícias e do sistema de Justiça, concentrando os esforços no aprisionamento em massa como modo de conter os índices de violência (Lum \& Koper, 2017).

Essa crise estimulou o surgimento de programas e pesquisas inovadoras de avaliação de impacto, resultando o que se denomina de SPBE, uma concepção que defende que os programas de prevenção à violência devem ser formulados utilizando as melhores evidências científicas disponíveis acerca do que funciona e do que não funciona, reduzindo a influência de crenças, interesses e preconceitos, provocando uma melhoria substancial na efetividade, na eficiência e na legitimidade das políticas públicas voltadas a reduzir a violência (Sherman, 1998; Welsh \& Farrington, 2012).

Os resultados das primeiras pesquisas de avaliação de impacto ${ }^{1}$, realizadas nos anos 1970, demonstraram que programas e crenças que tradicionalmente eram utilizados, como a colocação de tutores para disciplinar jovens infratores (Cabot, 1940), o aumento do número de viaturas fazendo patrulhamento (Kelling, Pate, Dieckman \& Brown, 1974), a redução do tempo de resposta aos chamados de emergência (Bieck \& Kessler, 1977; Spelman \& Brown, 1981) ou aulas dadas por policiais sobre os perigos do álcool e das drogas ilícitas (Ennet et al., 1994), não produziam impacto positivo e, algumas vezes, até mesmo pioravam a situação.

Por outro lado, estratégias inovadoras de prevenção à violência começaram a ser desenvolvidas, usando conhecimento científico sobre fatores de risco e padrões da dinâmica criminal, tornando as ações de prevenção à violência mais proativas e focadas, resultando na queda nos índices de violência e no aumento da eficiência dos gastos públicos, por serem muito mais baratas e efetivas do que as ações tradicionais de Justiça Criminal e encarceramento, como, por exemplo, o desenvolvimento de habilidades socioemocionais na primeira infância (Weikart, Deloria, Lawser \& Wiegerink, 1970), o policiamento em pontos quentes (Sherman \& Weisburd, 1995), a dissuasão focada sobre lideranças violentas (Kennedy, 1997), as mudanças na urbanização (Newman \& Franck, 1982), além de metodologias cognitivas comportamentais com presos altamente violentos (Andrews et al., 1990).

Conforme o número de evidências foi crescendo, também se tornou necessário sistematizar essas evidências para que se conseguisse entregar respostas mais sintéticas e com maior confiabilidade aos tomadores de decisão sobre o que efetivamente funciona. Dessa forma, a área de prevenção à violência começou a utilizar as revisões sistemáticas, um método científico que já vinha sendo muito utilizado na área da saúde, voltado a reunir de modo estruturado, rigoroso e replicável o maior número possível de estudos existentes sobre determinado tipo de problema e, assim, produzir uma evidência mais forte do que estudos isolados, sendo tais revisões consideradas atualmente o melhor

\footnotetext{
${ }^{1}$ As avaliações de impacto são um tipo de avaliação de resultado das políticas públicas, voltado a mensurar a efetividade de um programa, isto é, o quanto aquela ação específica alterou ou não determinado indicador no grupo que recebeu a intervenção em comparação com outro grupo semelhante que não recebeu a ação, podendo ser realizado de 2 formas: a) experimental, quando as unidades que receberão a intervenção e as que servirão de controle são escolhidas de forma randômica antes da aplicação do programa; b) quase experimental, quando as unidades de análise não foram escolhidas antes da aplicação do programa e são utilizadas técnicas estatísticas para avaliar o impacto do programa (Ramos \& Schabbach, 2012; Ramos, 2013).
} 
nível de evidência para a tomada de decisões em políticas públicas (Kugley et al., 2016; Lipsey, 2009; Weisburd, Farrington \& Gill, 2017).

Um importante marco histórico do amadurecimento da SPBE foi a realização de uma grande revisão sistemática, a pedido do Congresso dos Estados Unidos da América (EUA), por um grupo de renomados pesquisadores, com o objetivo de sistematizar o conhecimento que havia sido produzido até aquele momento, em língua inglesa, sobre o que funcionava e o que não funcionava para reduzir a violência, que ficou conhecida como Relatório Maryland (Sherman et al., 1997).

Os pesquisadores localizaram mais de 500 estudos que mensuraram o impacto de diferentes programas sobre comportamento violento, crimes violentos e reincidência criminal que atingiram os critérios técnicos estabelecidos no trabalho. Ao apresentar suas conclusões sobre o que funcionava e o que não funciona, o estudo teve grande repercussão em nível mundial ao longo dos anos seguintes, provocando mudanças na forma de formular e implementar programas de segurança pública, em diversos países do mundo.

Além disso, esse relatório estabeleceu os padrões utilizados até hoje em todo o mundo para avaliar a qualidade de avaliações de impacto e produzir sínteses de evidências, utilizando uma escala científica que ficou conhecida como Escala Maryland (Weisburd et al., 2017)2 .

Desde então, tanto a produção de avaliações de impacto quanto de revisões sistemáticas sobre a efetividade de programas na área de prevenção a violência vem aumentando em todo o mundo, chegando a uma média de 50 avaliações ao ano, entre 2007 e 2013 (Huges et al., 2014), e 12 revisões sistemáticas por ano, que já totalizam 474 estudos desse tipo publicados (Waar, 2019).

Como diversos autores têm apontado, o Brasil ficou alheio a esse avanço técnico da área de segurança, trazendo muitos prejuízos para a qualificação da área.

Segundo L. E. Soares (2006), a ausência de um debate cientificamente orientado sobre soluções faz com que as discussões sobre segurança pública permaneçam orientadas apenas por polarizações ideológicas e não por orientações técnicas, sem a preocupação de avaliar de modo rigoroso o que é feito.

Rolim (2006) destaca que, enquanto na Inglaterra e em outros países desenvolvidos a cultura do raciocínio especulativo e experimental fez com que, nas últimas décadas, tenha ocorrido uma revolução na criminologia, com a realização de pesquisas experimentais, o Brasil permaneceu preso a uma "pré-história da segurança pública", pela ausência do uso de evidências científicas na área, com as decisões sendo embasadas em crenças, culturas institucionais arcaicas, interesses privados e preconceitos.

Cano (2012) destaca que o conhecimento sobre avaliação de impacto foi relegado a segundo plano na formação das ciências sociais no Brasil, onde enfrenta uma estigmatização ideológica sobre o uso de métodos quantitativos mais complexos, provocando a ausência de produção desse tipo de conhecimento no país, o que acaba por retardar a avaliação e a qualificação das políticas nas mais diferentes áreas sociais, como educação, assistência e segurança.

Beato e Silveira (2014) destacam que a ausência de uma cultura de avaliação sobre programas de prevenção à violência dessa cultura de estudos faz com que não se consiga avançar na identificação das relações causais sobre o que funciona para reduzir os índices de violência, mantendo-se a ideia

${ }^{2}$ A referida escala será explicada adiante neste artigo. 
de que políticas sociais gerais ou, então, programas muito abrangentes e genéricos de segurança serão capazes de reduzir a violência.

A falta de produção de evidências de efetividade no país também tem sido destacada por diversas revisões não sistemáticas realizadas no país, que analisaram grande número de projetos já realizados no país (Beato \& Silveira, 2014; Cano et al., 2016; Muggah \& Aguirre, 2013; Willadino et al., 2011).

Não encontramos nenhuma revisão sistemática sobre a avaliação de efetividade de programas no Brasil, o que comprova o resultado já indicado por Abt e Winship (2016) em sua revisão mundial, que não localizou nenhum trabalho desse tipo em toda a América Latina.

Revisões Sistemáticas com outros enfoques já foram realizadas no país sobre o tema da violência, abordando temas como fatores de risco à violência (Murray, Castro Cerqueira \& Kahn, 2013), características da violência escolar (Nesselo et al., 2014), a relação entre violência na adolescência e cortisol (Lugarinho, Avanci \& Pinto, 2017), diversas perspectivas sobre causas e consequências da violência contra a mulher (Marasca et al., 2013; Patias, Bossi \& Dell’Aglio, 2014; Sá \& Werlang, 2013; Schein \& Martins, 2013; L. E. L. D. Silva \& Oliveira, 2015; Souza, 2016), a prevalência e as características da violência contra a pessoa idosa (Clementino, Marcolino \& Oliveira, 2013; Espíndola \& Blay, 2007; Minayo, Souza \& Paula, 2010).

Revisões sistemáticas mundiais localizaram um número muito pequeno de avaliações de impacto já produzidas no país. Uma revisão sistemática global de avaliações de efetividade de programas de policiamento em países em desenvolvimento localizou apenas 5 avaliações realizadas em tais países, das quais somente 1 no Brasil (Higginson, Mazerolle,Sydes, Davi \&s Mengersen, 2015).

\section{METODOLOGIA}

Para realizar esta revisão sistemática, foram utilizados, como base metodológica, os requisitos propostos pelo Manual da Cambpell Colaboration (Kugley et al., 2016). A inclusão e a sistematização das evidências foram realizadas segundo os critérios propostos pelo Relatório Maryland (Sherman, 1998), com o auxílio do Manual para Classificação de Métodos do Centro de Evidências para Desenvolvimento Econômico Local do Governo Inglês (Madaleno \& Waights, 2015), que auxiliou na análise da qualidade dos métodos quase-experimentais mais atuais.

O processo de busca e seleção foi posto em prática pelo coordenador da pesquisa e 2 assistentes de pesquisa, seguindo as evidências para reduzir os erros de busca e de digitação (Edwards et al., 2002). Ao final de cada etapa foram realizadas reuniões de sistematização e as discordâncias sempre foram resolvidas pelo coordenador da pesquisa.

\subsection{Critérios de inclusão}

Para responder à pergunta de pesquisa "Quais são as evidências existentes no Brasil sobre o que funciona para reduzir homicídios, roubos e estupros?” e definirmos com transparência os critérios de inclusão dos estudos, com o menor enviesamento possível, utilizamos o roteiro do acrônimo PICOS (Richardson et al., 1995), usualmente utilizado em revisões sistemáticas. No presente artigo, apresentaremos apenas os resultados relativos a homicídios.

- Participantes: as unidades de análise (pessoas, territórios, escolas, cidades, estados etc.) que receberam os efeitos da intervenção devem estar em território brasileiro; 
- Intervenção: qualquer tipo de intervenção (programas sociais, prevenção através do desenvolvimento pessoal, policiamento, prevenção situacional, justiça criminal, reabilitação ou outras), implementada por novas leis, planos, políticas, programas ou projetos desenvolvidos por agências públicas e/ou privadas no Brasil;

- Controle: os estudos devem ter utilizado um grupo de controlo, construído a partir da aleatorização, no caso de Experimentos, ou pareado, controlado estatisticamente e comparável nas suas principais variáveis antes da intervenção, no caso de estudos quase-experimentais;

- Outcome (Resultado): o estudo deve ter medido o desfecho da intervenção sobre homicídios, roubos e estupros, com dados de algum banco de dados oficial, em qualquer uma de suas formas relatadas, como, por exemplo, homicídio doloso, latrocínio, feminicídio, morte provocada pela polícia;

- Study Design (Desenho metodológico dos estudos): o estudo deve ter utilizado o método experimental ou quase experimental para comparar a situação de um grupo que recebeu a intervenção de outro grupo que não recebeu a intervenção, atingindo pelo menos o nível 3 da Escala Maryland. Para auxiliar na avaliação da qualidade dos métodos adotados nos estudos quase experimentais, seguimos os critérios propostos por Madaleno e Waights (2015).

Foram elegíveis estudos publicados ou não publicados, incluindo estudos relatados em artigos científicos, teses, dissertações e relatórios de pesquisa, elaborados em qualquer língua e sem restrição temporal.

Também foram incluídos estudos que analisaram o impacto do orçamento da segurança pública, do aumento do efetivo das polícias e do aumento do encarceramento, pois consideramos que esses temas são resultados de decisões políticas, portanto, aqui, são considerados intervenções. Também incluímos avaliações de programas que não tinham como meta declarada a redução de crimes violentos, mas que tiveram seu impacto avaliado sobre esses desfechos, seguindo a metodologia de Cano et al. (2016).

Após a constatação de que um estudo preencheu os critérios de inclusão, os diferentes módulos existentes dentro de cada estudo foram identificados. O módulo de um estudo é definido como cada um dos desfechos analisados (homicídios, roubos ou estupros) em relação a cada uma das diferentes intervenções avaliadas pelo estudo. Assim, é possível que um único estudo tenha analisado o efeito de 2 intervenções diferentes sobre os homicídios e sobre os roubos, sendo possível extrair, nesse exemplo, 4 módulos com apenas 1 estudo incluído, os quais serão extraídos e analisados separadamente, na planilha de resultados.

\subsection{Critérios de exclusão}

Foram excluídos estudos sobre: a) prevalência de violência; b) determinantes da violência, que tenham mensurado o impacto de fatores econômico, sociais e demográficos sobre os índices de violência; c) estudos de custo-benefício; d) fatores de risco; e e) causas da violência. Também foram excluídos estudos que avaliaram o impacto de programas de prevenção à violência, mas que mensuraram seus resultados em desfechos que não homicídios, roubos e estupros, como aqueles que mensuraram seus resultados sobre comportamento violento, rendimento escolar, violência escolar, consumo de álcool e outras drogas. 


\subsection{Estratégia de busca}

Diversas estratégias foram empregadas para realizar uma busca exaustiva de estudos que preenchessem os critérios de inclusão. Foram realizadas buscas avançadas em 9 portais eletrônicos de trabalhos científicos, em março e abril de 2018, nos seguintes portais nacionais: a) Periódicos da Coordenação de Aperfeiçoamento de Pessoal de Nível Superior (Capes); b) Scientific Electronic Library Online (SciELO); c) Biblioteca Virtual sobre Violência e Saúde (BVS/VS); d) Catálogo de Teses e Dissertações da Capes. Foram consultados os seguintes portais internacionais: a) PubMed; b) Education Resources Information Center (ERIC); c) PsycINFO; d) International Bibliography of the Social Sciences (IBSS); e) International Initiative for Impact Evaluation. E recorremos, ainda, às seguintes plataformas de busca: Google e Google Scholar.

A busca foi realizada por meio das seguintes palavras-chave: a) em português - policiamento, prevenção, tecnologia, segurança pública, guardas municipais, violência, crime, criminalidade, homicídio, morte violenta, crimes violentos letais intencionais, roubo, estupro, avaliação de impacto, avaliação de programa; b) em inglês - Brazil, policing, crime prevention, violence, crime, homicides, robbery, thefts, robberies, burglary, recidivism, program impact evaluation, experiments.

Para cada portal foi elaborada uma estratégia de busca específica, conforme suas regras internas de uso de conectores booleanos, hierarquização e organização dos estudos (Kugley et al., 2016). Em relação ao Google e ao Google Scholar, cada um dos pesquisadores realizou buscas com diferentes frases predefinidas, abrangendo os descritores da pergunta de pesquisa, dentro de um limite de até 400 resultados por busca.

Realizou-se, ainda, um grande esforço de busca manual, analisando todos os artigos publicados nos seguintes sites: a) Fórum Brasileiro de Segurança Pública e Revista do Fórum Brasileiro de Segurança Pública; b) Instituto Sou da Paz; c) Instituto Igarapé; d) Centro de Estudo de Segurança e Cidadania (CESeC); e) Centro de Estudos de Criminalidade e Segurança Pública (Crisp); f) Instituto de Pesquisas Econômicas Aplicadas (IPEA); g) Banco Nacional de Desenvolvimento Econômico e Social (BNDES); e h) Relatórios do Programa Pensando a Segurança, do Ministério da Justiça.

Para complementar a revisão e seguindo as recomendações da Campbell Collaboration (Kugley et al., 2016), realizou-se uma abrangente busca ativa, por meio do envio de e-mails para mais de 400 especialistas membros do Fórum Brasileiro de Segurança Pública, para os 143 grupos de pesquisa sobre segurança e violência das universidades brasileiras cadastrados no Portal da Capes, para 50 organizações não governamentais, para diversos organismos internacionais, para órgãos federais e estaduais de segurança pública e para os tribunais de contas da União e dos estados.

Além disso, realizou-se uma busca nas referências bibliográficas de todos os estudos incluídos e um e-mail foi enviado para os autores dos artigos incluídos, solicitando a indicação de outros estudos que pudessem ser analisados.

\subsection{Síntese dos dados}

O processo de síntese das evidências seguiu a metodologia proposta no Relatório Maryland (Sherman, 1998), a qual se divide em 2 etapas: a) análise individual da qualidade dos estudos; e b) produção da síntese das evidências. 


\subsubsection{Análise individual dos estudos identificados pela revisão sistemática}

Cada um dos estudos identificados no processo de revisão e pré-selecionados como avaliações de impacto sobre indicadores de homicídios, roubos e estupros foi analisado de acordo com sua capacidade para demonstrar a relação causa-efeito (validade interna), como ilustra o Quadro 1 (Sherman et al., 1997).

\section{QUADRO 1 PRIMEIRA ETAPA DA ESCALA MARYLAND - FORÇA PROBATÓRIA DE CAUSA E EFEITO}

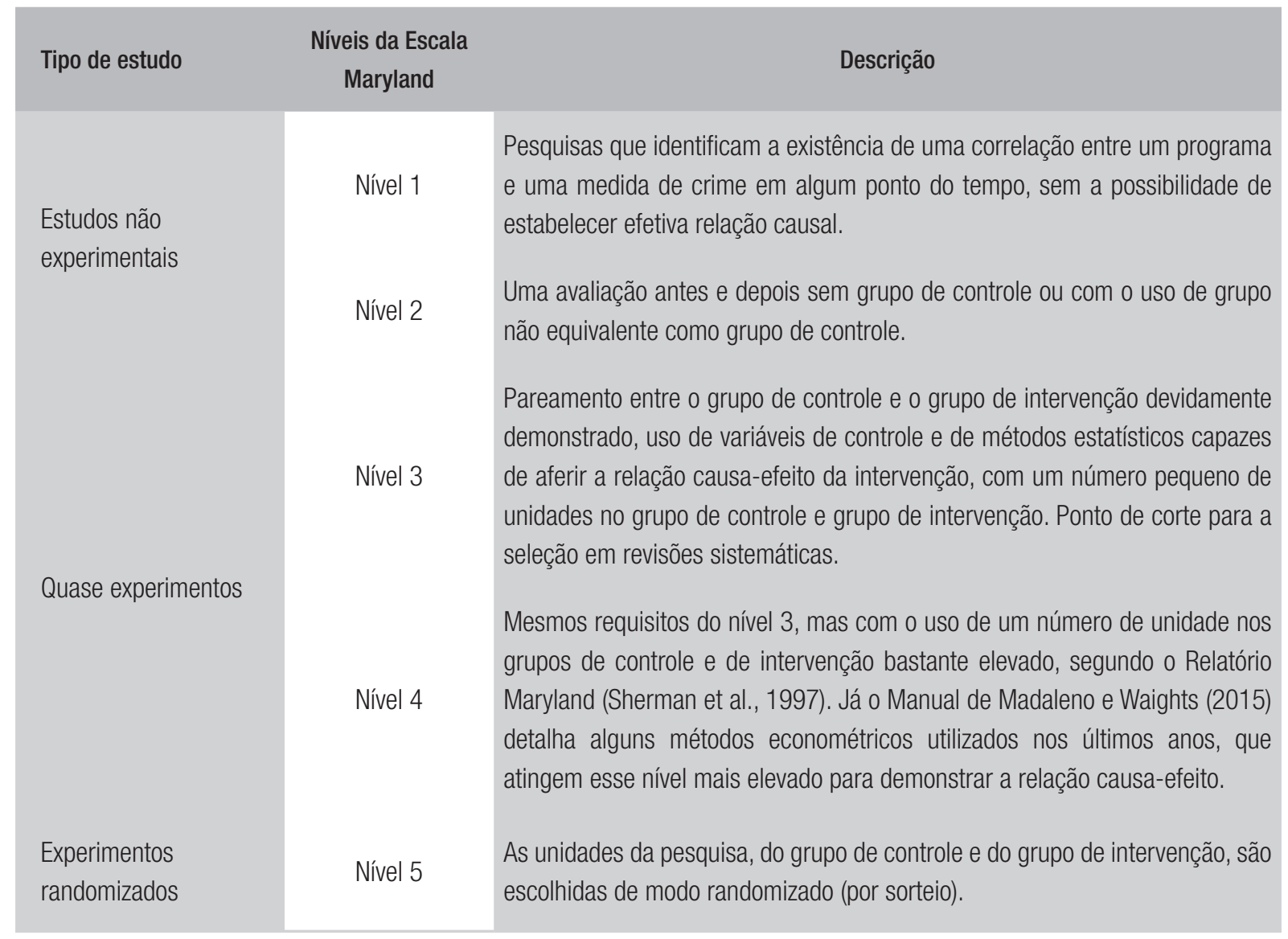

Fonte: Elaborado pelos autores com base em Sherman et al. (1997).

Cada um dos módulos dos estudos classificados acima do nível 3 foi classificado como: a) positivo, quando a intervenção produziu uma queda nos indicadores de violência, com significância em $p<0,1$ - o que equivale dizer que a probabilidade dos resultados encontrados no estudo se repetirem em outras situações é de 90\% (Ramos, 2014) -, como comumente utilizado na literatura da área (Lipsey, 2009); b) negativo, quando a intervenção produziu um aumento nos indicadores de violência $(\operatorname{com} p<0,1)$ e; c) sem impacto, quando a intervenção não resultou em impacto estatisticamente significativo. 
RAP | 0 que funciona e o que não funciona para reduzir homicídios no Brasil: uma revisão sistemática

\subsubsection{Produção da síntese de evidências}

Após o processo de inclusão dos estudos e a análise de cada um dos módulos desses estudos, os diferentes módulos foram agrupados em sínteses de evidências sempre que foi possível identificar que as características principais das intervenções analisadas eram semelhantes.

Por fim, as sínteses de evidências foram classificadas seguindo os critérios estabelecidos na segunda parte da Escala Maryland, como ilustra o Quadro 2 (Sherman et al., 1997).

\section{QUADRO 2 SEGUNDA ETAPA DA ESCALA MARYLAND - ESCALA PARA DEFINIÇÃO SOBRE 0 QUE FUNCIONA}

\begin{tabular}{|c|c|}
\hline Síntese de evidencia & Condições \\
\hline 0 programa funciona & $\begin{array}{l}\text { Devem ter pelo menos } 2 \text { avaliações de nível } 3 \text { com testes de significância estatística mostrando a } \\
\text { efetividade do programa. }\end{array}$ \\
\hline $\begin{array}{l}0 \text { programa é } \\
\text { promissor }\end{array}$ & $\begin{array}{l}\text { Quando o programa tem uma evidência positiva de pelo menos } 1 \text { estudo acima do nível três e a } \\
\text { preponderância nos demais estudos não experimentais. }\end{array}$ \\
\hline $\begin{array}{l}0 \text { programa não } \\
\text { funciona }\end{array}$ & $\begin{array}{l}\text { Quando existem evidências negativas em pelo menos } 2 \text { estudos acima do nível 3, com testes de } \\
\text { significância estatística mostrando a inefetividade do programa, demonstrando que o programa não } \\
\text { produz mudança significativa, e preponderância de não efetividade nos demais estudos de avaliação } \\
\text { de impacto excluídos. }\end{array}$ \\
\hline $\begin{array}{l}\text { Resultados } \\
\text { indefinidos }\end{array}$ & $\begin{array}{l}\text { Quando os estudos apresentam resultados significativos em sentidos opostos, alguns indicando } \\
\text { resultado estatisticamente significativo positivo e outros não. }\end{array}$ \\
\hline
\end{tabular}

Fonte: Elaborado pelos autores com base em Sherman et al. (1997).

\section{RESULTADOS}

\subsection{Resultados das buscas}

De um total inicial de 13.353 estudos rastreados e analisados, ao final, a revisão incluiu 41 estudos, seguindo o processo apresentado no fluxograma da Figura 1 (Moher, Liberati, Tetzlaff \& Altman, 2009). 


\section{FIGURA 1 FLUXOGRAMA DA INFORMAÇÃO DA REVISÃO SISTEMÁTICA}

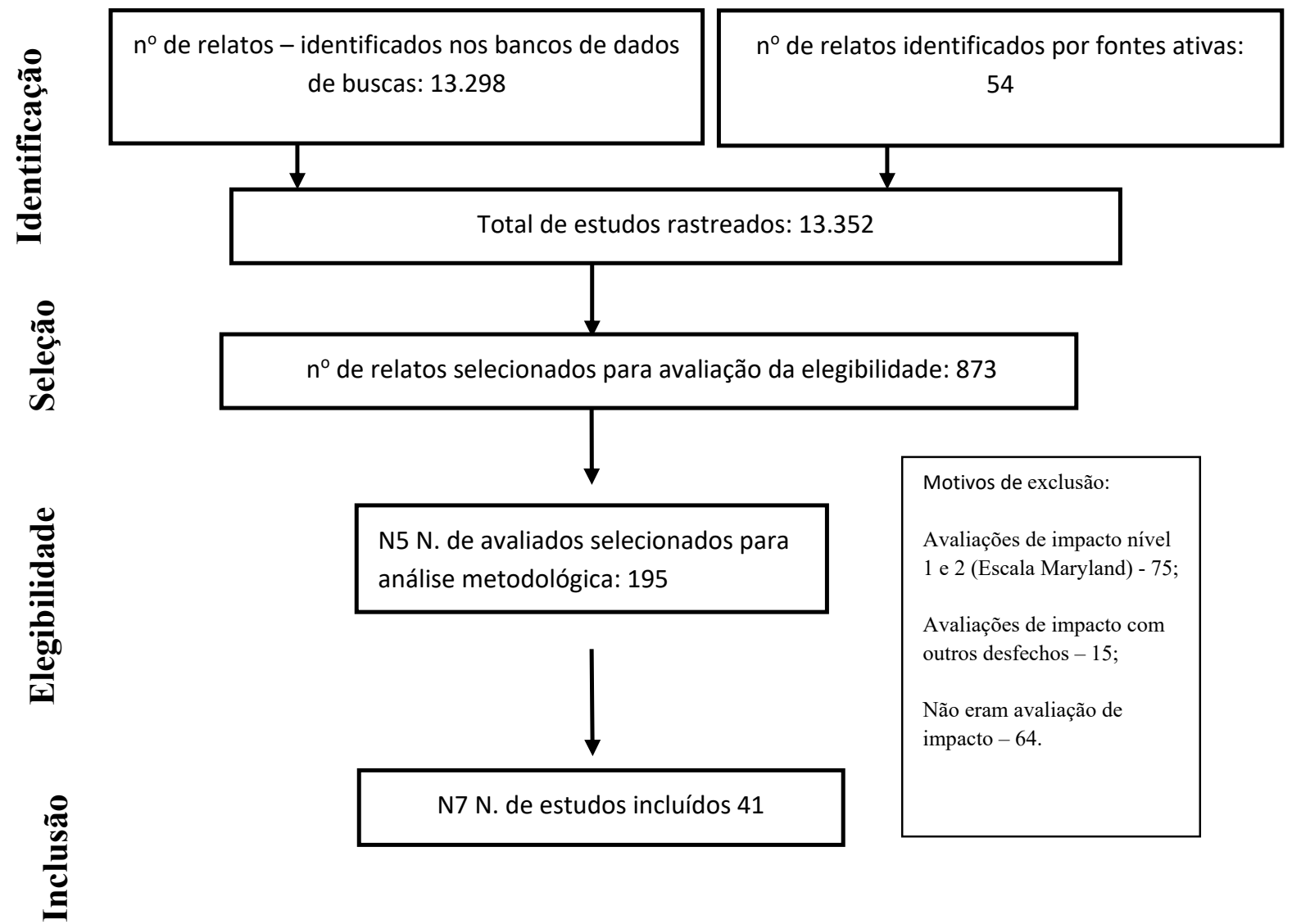

Fonte: Elaborada pelos autores com base em Moher et al. (2009).

Os 41 estudos incluídos resultaram em 86 módulos, conforme as características destacadas na tabela 1. 
RAP | 0 que funciona e o que não funciona para reduzir homicídios no Brasil: uma revisão sistemática

\section{TABELA 1 CARACTERÍSTICAS DOS ESTUDOS E MÓDULOS INCLUÍDOS NA REVISÃO SISTEMÁTICA}

\begin{tabular}{|c|c|c|c|c|c|}
\hline & $\mathrm{N}$ & $\%$ & & $\mathrm{~N}$ & $\%$ \\
\hline Tipo de publicação ( $\mathrm{n}=41$ ) & & & Desenho $(n=41)$ & & \\
\hline Artigo & 27 & 66 & Experimental & 0 & 0 \\
\hline Dissertação/tese & 11 & 27 & Quase experimental & 41 & 100 \\
\hline Relatório & 3 & 7 & Nível da Escala Maryland $(n=41)$ & & \\
\hline Ano de publicação $(n=41)$ & & & Nível 5 & 0 & 0 \\
\hline $1995-2000$ & 0 & 0 & Nível 4 & 5 & 12 \\
\hline 2001-2005 & 5 & 12 & Nível 3 & 35 & 88 \\
\hline $2006-2010$ & 8 & 20 & & & \\
\hline 2011-2015 & 17 & 41 & Tipo de intervenção $(n=85)$ & & \\
\hline \multirow[t]{2}{*}{$2016-2018$} & 11 & 27 & Gestão & 5 & 6 \\
\hline & & & Policiamento & 42 & 50 \\
\hline Idioma $(n=41)$ & & & Prevenção social & 1 & 1 \\
\hline Português & 30 & 73 & Prevenção situacional & 11 & 13 \\
\hline \multirow[t]{2}{*}{ Inglês } & 11 & 27 & Justiça Criminal & 15 & 17 \\
\hline & & & Política social & 6 & 7 \\
\hline Área dos autores $(n=41)$ & & & Mistos & 5 & 6 \\
\hline Economia & 38 & 94 & & & \\
\hline Ciências sociais & 1 & 2 & Desfecho $(n=85)$ & & \\
\hline Ciência política & 1 & 2 & Homicídio & 65 & 77 \\
\hline \multirow[t]{2}{*}{ Administração } & 1 & 2 & Roubo & 19 & 22 \\
\hline & & & Misto & 1 & 1 \\
\hline $\begin{array}{l}\text { Local onde a intervenção foi aplicada } \\
(n=41)\end{array}$ & & & Estupro & 0 & 0 \\
\hline País & 9 & 22 & & & \\
\hline Estado & 27 & 66 & Resultado & & \\
\hline \multirow[t]{3}{*}{ Município } & 5 & 12 & Funciona & 52 & 60 \\
\hline & & & Não impacto & 29 & 35 \\
\hline & & & Piora & 4 & 5 \\
\hline
\end{tabular}

Fonte: Elaborada pelos autores.

Nota: Em relação ao ano de 2018, só foram incluídos estudos divulgados até abril, quando esta revisão foi realizada.

Ao final, a síntese de evidências chegou aos resultados apresentados no Quadro 4. 


\section{QUADRO 4 O QUE FUNCIONA E 0 QUE NÃO FUNCIONA PARA REDUZIR HOMICÍDIOS NO BRASIL}

\begin{tabular}{|c|c|}
\hline & Homicídios \\
\hline Funciona & $\begin{array}{l}\text { Programas de Gestão Por Resultados (Cabral, 2016; Cerqueira, Coelho \& Silva, 2018; Soares \& Viveiros, 2010); } \\
\text { Restrição do horário de venda de bebida alcoólica (Biderman, Mello \& Schneider, 2009; Schneider, 2005; } \\
\text { Scripilliti, 2006); } \\
\text { Implantar Guarda Municipal (Cabral, 2016; Ferreira, 2012; Pereira, 2016; Scripilliti, 2006); } \\
\text { Programa Fica Vivo (Castro, 2014; Peixoto, Andrade \& Azevedo, 2008a, 2008b; Silva et al., 2016); } \\
\text { UPP - autos de resistência (Butelli, 2015; Ferraz \& Ottoni, 2013; Magaloni, Franco, Melo, 2015); } \\
\text { Estatuto do Desarmamento (Cerqueira, 2013; Cerqueira \& Mello, 2013); } \\
\text { Lei Maria da Penha (Azuaga \& Sampaio, 2017; Cerqueira et al., 2015; Schiavon, 2017); }\end{array}$ \\
\hline Promissor & $\begin{array}{l}\text { Performance do Poder Judiciário (Abras, Araújo \& Shikida, 2014). } \\
\text { Criar Secretaria Municipal de Segurança (Scripilliti, 2006); } \\
\text { Disque-Denúncia (Scripilliti, 2006); } \\
\text { Policiamento com foco em armas (Oliveira \& Rostirolla, 2017); } \\
\text { Programa de abertura de escolas nos finais de semana (Vasconcellos \& Menezes, 2005); } \\
\text { Eletrificação/lluminação (Arvate, Falsete, Ribeiro \& Souza, 2018) } \\
\text { Juizado de Violência Doméstica e Familiar contra a mulher (Schiavon, 2017) } \\
\text { Aumentar o orçamento da assistência social (Loureiro \& Carvalho, 2006) } \\
\text { UPP - homicídio doloso (Butelli, 2015; Ferraz \& Ottoni, 2013) }\end{array}$ \\
\hline Indefinido & $\begin{array}{l}\text { Efetivo Policial (Fajnzylber \& Araújo, 2001; Pereira, 2016; Sachsida \& Mendonça, 2013, 2014; Schuch, 2017); } \\
\text { Orçamento da Segurança Pública (Kume, 2004; Loureiro \& Carvalho, 2006; Pereira, 2016; Santos, 2009); } \\
\text { Delegacia da Mulher (Perova \& Reynolds, 2016; Schiavon, 2017) } \\
\text { Campanha de entrega de armas (Scorzafave, Soares \& Dorigan, 2015); } \\
\text { Lei de Drogas de } 2006 \text { (Sachsida \& Mendonça, 2014); } \\
\text { Redução da maioridade (Costa et al., 2018). }\end{array}$ \\
\hline $\begin{array}{l}\text { Não produz } \\
\text { impacto }\end{array}$ & $\begin{array}{l}\text { Programa Bolsa Família (Chioda, Mello \& Soares, 2016; Loureiro, 2012); } \\
\text { Encarceramento (Gaulez, Ferro \& Moreira, 2018; Sachsida \& Mendonça, 2013, 2014; Schuch, 2017). }\end{array}$ \\
\hline
\end{tabular}

Fonte: Elaborado pelos autores.

\subsection{Descrição das evidências que funcionam}

\subsubsection{Gestão por resultado}

O uso intensivo de análise criminal e o modelo de gestão Compstat serviu de inspiração para diversas experiências inovadoras de gestão de estados e municípios na área de segurança no Brasil. Apesar desses programas terem sido compostos por diversas outras iniciativas, todos se basearam no uso intensivo de análise criminal sobre territórios integrados entre Polícia Militar e Polícia Civil, com forte participação da liderança política. 
A revisão localizou um total de 13 avaliações de impacto de programas de gestão por resultado em relação a homicídios já feitos no Brasil, sendo que 10 avaliações não foram incluídas por não preencherem os critérios de inclusão, e a grande maioria apontou que os programas produziram resultados positivos: a) Projeto Polícia de Resultados de Minas Gerais (Beato et al., 2001; Loschi et al., 2005); b) Sistema Integrado de Monitoramento do Rio de Janeiro (Cabral, 2016; Campagnac \& Ferreira, 2013); e c) Pacto pela Vida de Pernambuco (Oliveira, 2016; Penha et al., 2013; Ratton \& Daudelin, 2018; B. F. A. Silva et al., 2016; Zaverucha \& Nóbrega, 2015; Silveira Neto et al., 2014).

Os 3 estudos incluídos avaliaram 3 diferentes programas de gestão por resultados, quais sejam: a) o Programa Integração da Gestão em Segurança Pública (IGESP), de Minas Gerais (R. Soares \& Viveiros, 2010); b) o Infocrim, de São Paulo (Cabral, 2016); e c) o Programa Estado Presente, do Espírito Santo (Silva, Cerqueira \& Coelho, 2018). Eles concluíram que esse tipo de programa produziu uma queda no número de homicídios, entre $9 \%$ e 17\%, sendo que em R. Soares e Viveiros (2010) o resultado não apresentou significância estatística, levando os programas de gestão por resultado a serem classificados como "funciona" para reduzir homicídios.

\subsubsection{Programa Fica Vivo!}

O Programa Fica Vivo! nasceu em 2001, por meio de uma iniciativa do Centro de Estudos em Criminalidade e Segurança Pública da Universidade Federal de Minas Gerais (CRISP/UFMG) (Beato et al., 2001), sendo composto por ações de repressão focada em territórios com altos índices de homicídios, combinando dissuasão sobre indivíduos violentos, policiamento de proximidade, programas de prevenção social para jovens em situação de risco e mobilização comunitária, mantendo-se em funcionamento até hoje; atende, anualmente, mais de 12.500 jovens em situação de risco em 32 comunidades (Souza, 2016). A revisão localizou 9 avaliações de impacto do Programa Fica Vivo!, sendo que 5 não preencheram os critérios de inclusão (Alves \& Arias, 2012; Higginson et al., 2015; Matta \& Andrade, 2005; Silveira, 2008; Silveira, Assunção, Silva \& Beato, 2010) e 4 preencheram (Castro, 2014; Peixoto et al., 2008a, 2008b; B. F. A. Silva et al., 2016), as quais concluíram que o programa produz uma redução substancial de homicídios nas áreas que o receberam, estimada em $43 \%$ do total de homicídios observados nas áreas onde o programa atuou entre 2005 e 2015 (B. F. A. Silva et al., 2016) ou de 20 a 23 homicídios por 100 mil habitantes nas comunidades que receberam o programa (Peixoto et al., 2008b), e que esse resultado aumenta ao longo do tempo (Peixoto et al., 2008a; B. F. A. Silva et al., 2016).

\subsubsection{Restrição do horário de venda de bebida alcoólica}

No início dos anos 2000, diversos municípios da Região Metropolitana de São Paulo estabeleceram restrição de horário de venda de bebida alcoólica à noite. A revisão sistemática localizou 5 estudos de avaliação de impacto sobre essa experiência, sendo que 2 não preencheram os critérios de inclusão (Duailibi et al., 2007; Kahn \& Zanetic, 2005; Volpe, Ladeira \& Fantoni, 2017) e 3 preencheram os requisitos de inclusão (Biderman et al., 2009; Schneider, 2005; Scripilliti, 2006), os quais concluíram que a restrição provocou uma redução de $10 \%$ a $20 \%$ no número de homicídios, sendo que os autores destacaram que, além da aprovação da lei, a efetividade da fiscalização foi fator fundamental para o resultado. 


\subsubsection{Estatuto do Desarmamento}

A Lei n. 10.826 (Estatuto do Desarmamento, 2003) restringiu fortemente o comércio de armas de fogo no país e proibiu o porte para a grande maioria da população. A revisão sistemática localizou 9 avaliações de impacto do Estatuto do Desarmamento (2003), mas, no entanto, 7 delas não preencheram os critérios de inclusão (Ary, Soares, Cerqueira, 2015; Hartung \& Pessoa, 2009; Justus \& Kassouf, 2013; Kahn \& Zanetic, 2005; Souza et al., 2007; Organização das Nações Unidas para a Educação, a Ciência e a Cultura [UNESCO], 2005; Waiselfisz, 2015), sendo que a maioria desses trabalhos indicam que o estatuto produziu queda significativa no número de homicídios ou internações hospitalares no país. Dois estudos foram incluídos (Cerqueira, 2014; Cerqueira \& Mello, 2013) e concluíram que o Estatuto do Desarmamento (2003) provocou uma queda nos índices de homicídios, estimada por Cerqueira e Mello (2013) em torno de 12,5\%, sem provocar um aumento dos homicídios provocados por outros instrumentos.

\subsubsection{Policiamento de proximidade em comunidades de ocupação desordenada, socialmente vulneráveis e com forte presença de grupos criminosos - Unidades de Polícia Pacificadora (UPP)}

As unidades de polícia pacificadora (UPPs) foram um programa de segurança pública implementado pelo Governo do Estado do Rio de Janeiro, na preparação para a Copa do Mundo FIFA de 2014 e para os Jogos Olímpicos de 2016. Entre 2008 e 2014 foram instaladas 38 UPPs, com quase 10 mil policiais, atendendo a um total de 700 mil habitantes. A revisão encontrou 4 avaliações de impacto das UPPs, sendo que 1 estudo (Cano, 2012), que também encontrou reduções significativas nos níveis de letalidade policial, não preencheu os requisitos de inclusão estabelecidos, e 3 estudos foram incluídos (Butelli, 2015; Magaloni et al., 2015; Ottoni \& Ferraz, 2014), os quais identificaram que as UPPs provocaram uma expressiva redução no número de mortes provocadas pelas polícias, variando entre $60 \%$ e $78 \%$, que caíram de 1.300 pessoas mortas pela polícia, em 2007, para 400, em 2013. Magaloni et al. (2015) identificaram que o programa teve maior êxito em comunidades com menor nível de vulnerabilidade, em particular nos níveis de alfabetização e oportunidades de trabalho e em comunidades menores.

\subsubsection{Lei Maria da Penha}

A Lei n. 11.340 (Lei Maria da Penha, 2006), um marco legal voltado a organizar um sistema de prevenção à violência contra a mulher e uma rede de atendimento e proteção. A revisão localizou a existência de 3 avaliações de impacto sobre a Lei Maria da Penha (2006), sendo que os 3 estudos (Azuaga \& Sampaio, 2017; Cerqueira, Matos, Martins \& Pinto, 2015; Schiavon, 2017) concluíram que a Lei Maria da Penha (2006) e a implantação de suas medidas provocaram uma redução de 10\% a 19\% sobre os homicídios de mulheres no Brasil.

\subsubsection{Criação de Guardas Municipais}

A revisão localizou e incluiu 4 estudos que avaliaram o impacto da criação das guardas municipais sobre os crimes violentos, sendo que 2 avaliaram esse impacto apenas em São Paulo (Cabral, 2016; Scripilliti, 2006) e outros 2 em relação a todo o país (Ferreira, 2012; Pereira, 2016). Os 4 estudos encontraram que a criação de guardas municipais produziu uma redução do número de homicídios, 
sendo que Ferreira (2012) indicou que esse impacto foi de 15\%. O estudo de Pereira (2016) apontou que o tamanho do efetivo das guardas municipais não está relacionado ao seu resultado e também constatou que o efeito da criação foi maior nos municípios entre 20 e 99 mil habitantes.

\section{CONCLUSÃO}

Os resultados da revisão demonstraram que, embora incipiente no Brasil, já existe um conjunto expressivo e relevante de evidências científicas sobre o que funciona e o que não funciona para reduzir o número de homicídios, o que se mostra muito relevante para que o país possa construir políticas públicas eficientes para diminuir a violência, afastando visões baseadas em crenças, interesses, preconceitos ou populismo político.

A revisão identificou que, desde o estudo pioneiro (Fajnzylber \& Araujo, 2001), a produção de avaliações de impacto vem crescendo de modo acelerado desde 2012, demonstrando que o paradigma da SPBE está avançando no Brasil.

Embora o número de avaliações de impacto localizadas tenha sido relevante, os 41 estudos incluídos nessa revisão representam apenas $20 \%$ do total de estudos identificados pelo Relatório Maryland, em 1997, o qual incluiu um total de 204 avaliações de impacto sobre crimes violentos (Weisburd, Lum \& Petrosino, 2001), demonstrando que o país, apesar da gravidade do tema da violência e do volume de recursos investidos na área, ainda permanece bastante atrasado na produção de evidências sobre o que funciona.

Um dos dados mais negativos encontrados pela revisão recai sobre o fato de não ter sido localizado nenhum Experimento Randomizado Controlado sobre crimes violentos, enquanto que no Relatório Maryland foram incluídos 46 estudos desse tipo (Weisburd et al., 2001) e outra revisão, realizada em 2009, constatou que já foram conduzidos pelo menos 185 experimentos em todo o mundo, apenas na área de policiamento e Justiça Criminal (Telep, 2009). Embora sejam mais difíceis de ser realizados, pois exigem disponibilidade e conhecimento técnico por parte de tomadores de decisão e pesquisadores, os experimentos representam um passo fundamental para a política baseada em evidências, em razão da qualidade do conhecimento que produzem e do impacto na mudança dos rumos das políticas públicas (Weisburd et al., 2001).

Quanto à área de conhecimento dos autores, é importante notar a grande preponderância de autores na área da economia, que foram os primeiros autores de 38 dos 41 estudos (93\%). Esta informação comprova o argumento que tem sido destacado por vários autores no país em relação à resistência da utilização de métodos quantitativos nas ciências sociais no Brasil, o que enfraquece a construção de uma perspectiva de pluralismo metodológico fundamental para a melhoria das políticas públicas no país, incluindo na área da prevenção da violência (Beato, 2002; Rolim, 2006; Willadino et al., 2011; Marino, 2012; Beato \& Silveira, 2014; Cano et al., 2016; Ludmila \& Niche, 2018).

Outro resultado que merece destaque é o fato de três das principais crenças habitualmente citadas no debate político do Brasil como soluções para o problema da violência: o aumento do orçamento da Segurança Pública, o aumento do número de agentes policiais e o aumento do número de prisioneiros, não terem se revelado eficazes na redução dos homicídios. Estes dados demonstram que é necessário modificar a forma como a prevenção da criminalidade é entendida e feita no país, ainda ligada ao modelo tradicional de segurança pública, que se resume a ações reativas, sem foco e fragmentadas, visando o encarceramento. É importante salientar que estes resultados estão de acordo 
com as evidências internacionais que demonstram que o mero aumento das despesas, do pessoal e do número de presos não reduz as taxas de homicídios (Carriaga \& Worrall, 2015; Marvell \& Moody, 1996; Lum \& Koper, 2017).

Vale destacar que esses resultados vão ao encontro das evidências internacionais que têm sido produzidas desde os primórdios da SPBE, demonstrando que o mero aumento do gasto ou do efetivo não é capaz de reduzir os índices de violência, se forem empregados de modo reativo e sem foco (Carriaga \& Worrall, 2015; Lum \& Koper, 2017; Marvell \& Moody, 1996).

O conjunto de programas que funcionam tem algumas características em comum que podem auxiliar na formulação de novos programas efetivos no país e que seguem os princípios gerais dos programas efetivos em nível internacional (Abt \& Winship, 2016; Lum \& Koper, 2017; Kopittke, 2019). Em geral, eles são:

a) proativos, pois aumentam a capacidade de análise criminal e permitem uma atuação preventiva, antes dos crimes ocorrerem, como no caso dos programas de gestão por resultado;

b) altamente focados, seja em determinados territórios específicos com elevados índices de violência (como é o caso do Programa Fica Vivo! e das UPPs), em determinadas circunstâncias que aumentam o risco de violência, como a disponibilidade de armas (como o Estatuto do Desarmamento) e de álcool (como a experiência de regulação do horário de venda feita em São Paulo), ou em pessoas específicas que têm maior risco de vitimização, como no caso da Lei Maria da Penha (2006);

c) a integração de forças é outra característica que parece aumentar a efetividade dos programas, sendo que um número maior de instituições envolvidas e atuando de modo proativo e com o mesmo foco potencializam os resultados positivos.

Foi possível perceber um elevado nível de convergência entre os resultados encontrados pela revisão e os resultados que têm sido indicados pela literatura internacional, o que indica que o Brasil deve aproveitar, de modo mais intensivo as estratégias que já têm comprovação científica de que funcionam em nível internacional, sempre procurando identificar as peculiaridades locais que podem dificultar ou potencializar determinada estratégia.

Em relação à violência contra a mulher, no total 5 estudos, foram avaliados impactos de intervenções sobre feminicídios, o equivalente a $12,5 \%$ e identificou-se que equipamentos e políticas de proteção à mulher vítima de violência apresentam potencial para reduzir o número de mulheres assassinadas. No entanto, esse número é pequeno se comparado às 232 avaliações já produzidas em países desenvolvidos, muitas delas do tipo experimental num dos países em que mais mulheres são vítimas de violência em todo o mundo. É importante destacar que a utilização de estratégias mais focadas, integradas podem aumentar a efetividade das ações (Schucan-Bird, Vigurs, Quy \& Gough, 2016).

Com esses resultados, esta revisão demonstrou que o país já possui diversos tipos de programas com efetividade cientificamente comprovada e que as evidências nacionais possuem grande convergência com as evidências internacionais. Por esse motivo é de grande relevância que esse conhecimento seja utilizado nas decisões que são tomadas na área, seja pelo Poder Executivo, Judiciário ou Legislativo, com o objetivo de melhorar a efetividade, a eficiência e a legitimidade das medidas tomadas, fazendo os índices de violência serem reduzidos dentro dos marcos do Estado democrático de direito. 


\section{RECOMENDAÇÕES}

\subsection{Recomendações para as políticas}

Os governos, organismos internacionais e financiadores privados podem utilizar a sistematização de conhecimento baseado em evidências para orientar sua tomada de decisão, priorizando o financiamento de intervenções que tenham evidências científicas de sua efetividade, além de apoiarem novas avaliações daquelas que têm evidências promissoras e não investindo naquelas ações em que as evidências indicam que a intervenção não funciona (Lum, Koper \& Telep, 2011).

Outra recomendação de grande relevância, destacada no relatório Maryland (Sherman, 1998) e útil ao Brasil, é que as agências de financiamento internacional e o Governo Federal muito mais do que repassarem recursos, assumam um papel de protagonismo na produção e disseminação de evidências, auxiliando estados e municípios a promover uma mudança da qualidade das políticas desenvolvidas na área.

Também é de grande relevância que as casas parlamentares utilizem evidências no momento de formular as mais diferentes leis que possam ter impacto sobre os índices de violência, podendo construir centros de produção de evidências ou parcerias com centros de pesquisa que trabalhem com evidências.

Outro elemento de grande relevância para o Brasil seria utilizar a concepção de SPBE como o centro da matriz curricular do processo de formação de profissionais da área de segurança pública (Lum \& Koper, 2017), o que poderia ser uma iniciativa das academias de polícia e das guardas municipais no país.

Os órgãos de controle da administração pública, como tribunais de contas e Ministério Público, também podem impulsionar a disseminação do uso de evidências, estimulando que os governos justifiquem os programas que desenvolvem com base em evidências. Nesse sentido, seria muito relevante que o país aprovasse uma legislação tornando obrigatória a necessidade de justificar as políticas públicas com base em evidências.

As diferentes esferas de governo e as instituições também podem criar centros voltados à produção e disseminação de evidências, nos moldes do What Works Centre, do governo inglês, e também podem ser criadas plataformas on-line de evidências, capazes de traduzir e disseminar as evidências disponíveis de modo simples e prático.

\subsection{Recomendações para a pesquisa acadêmica}

A revisão demonstrou que, hoje, o esforço para a produção de evidências se encontra muito concentrado na área da economia, sendo de grande relevância que outras áreas com grande produção científica sobre o tema da violência e da segurança pública também se aproximem do paradigma da SPBE e passem a produzir avaliações de impacto e revisões sistemáticas sobre as mais diferentes intervenções, com o objetivo de aumentar sua capacidade de influenciar as políticas públicas.

Mostra-se de grande relevância que os pesquisadores de todas as áreas busquem desenvolver experimentos científicos para avaliar programas de prevenção à violência e que as agências de fomento, os órgãos públicos e os financiadores privados proporcionem recursos a esse tipo de iniciativa. 


\section{REFERÊNCIAS}

Abras, L. L. H., Araújo, A. F., Jr., Shikida, C. D., \& Shikida, A. P. F. A. (2014). Mais armas, menos crimes? Uma análise econométrica para o estado de Minas Gerais. Rev. Ciênc. Empres, 15(1), 5-24.

Abt, T., \& Winship, C. (2016). What works in reducing community violence: a meta-review and field-study for the northern triangle (USAID Report). Washington, DC: United States Agency for International Development.

Alves, M. C., \& Arias, E. D. (2012). Understanding the Fica Vivo programme: two-tiered community policing in Belo Horizonte, Brazil. Policing and Society, 22(1), 101-113.

Andrews, D. A., Zinger, I., Hoge, R. D., Bonta, J., Gendreau, P., \& Cullen, F. T. (1990). Does correctional treatment work? A psychologically informed meta-analysis. Criminology, 28, 369-404.

Arvate, P., Falsete, F. O., Ribeiro, F. G., \& Souza, A. P. (2018). Lighting and homicides: Evaluating the effect of an electrification policy in rural Brazil on violent crime reduction. J Quant Criminol, 34, 1047-1078.

Ary, G., Soares, D., \& Cerqueira, D. R. C. (2015). Estatuto do desarmamento: Um tiro que não saiu pela culatra. Insight - Inteligência, 68(17), 78-86.

Azuaga, F. L., \& Sampaio, B. (2017). Violência contra mulher: o impacto da Lei Maria da Penha sobre o feminicídio no Brasil. In Anais do 450 Encontro ANPEC, Natal, RN.

Beato, C. (2002). Crime and social policies in Latin America: problems and solutions. Washington, DC: Woodrow Wilson Center Update on the Americas.

Beato, C., Assunção, R. M., Silva, B. F. A. D., Marinho, F. C., Reis, I. A., \& Almeida, M. C. D. M. (2001). Conglomerados de homicídios e o tráfico de drogas em Belo Horizonte, Minas Gerais, Brasil, de 1995 a 1999. Cadernos de Saúde Pública, 17, 1163-1171.

Beato, C., \& Silveira, A. M. (2014). Efetividade e avaliação em programas de prevenção ao crime em Minas Gerais (Artigos Estratégicos). Rio de Janeiro, RJ: Instituto Igarapé.

Biderman, C., Mello, J. M., \& Schneider, A. (2009). Dry laws and homicides: evidence from the São Paulo metropolitan area. The Economic Journal, 120(543), 157-182.
Bieck, W., \& Kessler, D. A. (1977). Response team analysis. Kansas City, MO: Board of Police Commissioners.

Butelli, P. H. (2015). Avaliação de impacto de políticas de segurança: o caso das Unidades de Polícia Pacificadora (UPPS) no Rio de Janeiro (Tese de Doutorado). Fundação Getulio Vargas, São Paulo, SP.

Cabot, R. D. Q. (1940). A long-term study of children: the Cambridge-Somerville Youth Study. Child Development, 11, 143-151.

Cabral, M. V. F. (2016). Avaliação do impacto do Infocrim sobre as taxas de homicídios dos municípios paulistas: uma aplicação do método de diferenças em diferenças espacial (Tese de Doutorado). Universidade Federal de Juiz de Fora, Juiz de Fora, MG.

Campagnac, V., Ferreira, M. (2013). Avaliação do Impacto do Uso da Informação na Prevenção de roubo a transeunte no Estado do Rio de Janeiro. In Anais do 60 Congresso Consad, Rio de Janeiro, RJ.

Campos, M. S. (2010). Crime e Congresso Nacional no Brasil pós-1988: uma análise da política criminal aprovada de 1989 a 2006 (Dissertação de Mestrado). Universidade Estadual de Campinas, Campinas, SP.

Cano, I. (2012). Nas trincheiras do método: o ensino da metodologia das ciências sociais no Brasil. Sociologias, 14(31), 94-119.

Cano, I., Rojido, E., Chaves, R., Bezerra, J., \& Sá, B. (2016). Mapeamento de programas de prevenção de homicídios na América Latina e Caribe. Rio de Janeiro, RJ: Universidade do Estado do Rio de Janeiro.

Carriaga, M. L., \& Worrall, J. L. (2015). Police levels and crime. The Police Journal: Theory, Practice and Principles, 88(4), 315-333.

Castro, T. E. (2014). Avaliação de impacto do Programa Fica Vivo! sobre a taxa de homicídios em Minas Gerais (Dissertação de Mestrado). Universidade Federal de Viçosa, Viçosa, MG.

Cerqueira, D. (2014). Causas e consequências do crime no Brasil. Rio de Janeiro, RJ: Banco Nacional de Desenvolvimento Econômico e Social.

Cerqueira, D., Matos, M., Martins, A. P. A., \& Pinto, J., Jr. (2015). Avaliando a efetividade da Lei Maria da Penha (Texto para Discussão IPEA n. 2048). Brasília, DF: Instituto de Pesquisa Econômica Aplicada. 
Cerqueira, D., \& Mello, J. M. P. (2013). Evaluating a national anti-firearm law and estimating the causal effect of guns on crime (Texto para Discussão n. 607). Rio de Janeiro, RJ: Universidade Católica do Rio de Janeiro.

Chioda, L., Mello, J. M. P., \& Soares, R. R. (2012, fevereiro) Spillovers from Conditional Cash Transfer Programs: Bolsa Familia and Crime in Urban Brazil (Working Paper, 024). Rio de Janeiro, RJ: Rede de Economia Aplicada.

Clementino, F. S., Marcolino, E. C., \& Oliveira, K. K. D. (2013). Uma revisão sistemática acerca da violência doméstica contra idosos: conhecendo a vítima. In Anais do 30 Congresso Internacional do Envelhecimento Humano, Campina Grande, PB.

Costa, F. J. M., Faria, J. S., Iachan, F. S., \& Caballero, B. (2018, Fall). Homicides and the Age of Criminal Responsibility in Brazil: A Density Discontinuity Approach. Economia, 19(1), 59-62.

Duailibi, S., Ponicki, W., Grube, J., Pinsky, I., Laranjeira, R., \& Raw, M. (2007). The effect of restricting opening hours on alcohol-related violence. American Journal of Public Health, 97(12), 2276-2280.

Edwards, P., Clarke, M., DiGuiseppi, C., Pratap, S., Roberts, I., \& Wentz, R. (2002). Identification of randomized controlled trials in systematic reviews: accuracy and reliability of screening records. Statistics in Medicine, 21(11), 1635-1640.

Ennet, S. T., Rosenbaum, D. P., Flewelling R. L., Bieler, G. S., Ringwalt, C. L., \& Bailey, S. L. (1994). Long-term evaluation of drug abuse resistance education. Addictive Behaviors, 19(2), 113-125.

Espíndola, C. R., \& Blay, S. L. (2007). Prevalência de maus-tratos na terceira idade: revisão sistemática. Revista de Saúde Pública, 41, 301-306.

Fajnzylber, P., \& Araujo, A. F., Jr. (2001). O que causa a criminalidade violenta no Brasil? Uma análise a partir do modelo econômico do crime: 1981 a 1996 (Texto para Discussão Cedeplar n. 162). Belo Horizonte, MG: Universidade Federal de Minas Gerais.

Ferreira, L. R. C. (2012). O papel das guardas municipais na redução de homicídios: evidências empíricas para o Brasil (Dissertação de Mestrado). Fundação Getulio Vargas, São Paulo, SP.
Gaulez, M. P., Ferro, A. R., \& Moreira, G. C. (2018). $\mathrm{O}$ efeito do encarceramento de homicidas sobre a taxa de homicídios no Brasil. EALR, 9(2), 288-307.

Global Burden of Disease Collaborative Network. (2017). Global burden of disease study. Seattle, WA: Autor.

Hartung, G., \& Pessoa, S. (2009). O papel das armas de fogo na queda dos homicídios em São Paulo. In Anais do 370 Encontro ANPEC, Foz do Iguaçu, PR.

Higgins, J. P. T., \& Green, S. (Eds.). (2011). Cochrane handbook for systematic reviews of interventions. Hoboken, NJ: John Wiley \& Sons.

Higginson, A., Mazerolle, L., Sydes, M., Davis, J., \& Mengersen, K. (2015). Policing interventions for targeting interpersonal violence in developing countries: a systematic review (3ie Systematic Review, No. 20). London, England: International Initiative for Impact Evaluation.

Huges, K., Bellis, M., Hardcastle, K., Butchart, A., Dahlberg, L., Mercy, J., \& Mikton, C. (2014). Global development and diffusion of outcome evaluation research for interpersonal and self-directed violence prevention from 2007 to 2013: A systematic review. Aggress Violent Behav, 19(6), 655-662.

Instituto de Pesquisa Econômica Aplicada, \& Fórum Brasileiro de Segurança Pública. (2019). Atlas da violência 2019. Brasília, DF: Autor.

Justus, M., \& Kassouf, A. L. (2013). Evidence of the effect of wealth observed by criminals on the risk of becoming a victim of property crimes. Economia, 14, 88-101.

Kahn, T., \& Zanetic, A. (2005, julho). O Papel dos Municípios na Segurança Pública (Estudos Criminológicos, 4). São Paulo, SP: Observatório de Segurança Pública. Recuperado de http:// www.observatoriodeseguranca.org/files/ Papel\%20dos\%20munic\%C3\%ADpios\%20na\%20 Seguran\%C3\%A7a\%20P.pdf

Kelling, G. L., Pate, T., Dieckman, D., \& Brown, C. (1974). The Kansas City Preventive Patrol Experiment: a technical report. Washington, DC: Police Foundation.

Kennedy, D. M. (1997). Pulling levers: chronic offenders, high-crime settings, and a theory of prevention. Valparaiso University Law Review, 31, $449-484$. 
Kopittke, A. L. W. (2016). Segurança pública e democracia. Uma história de desencontros (Dissertação de Mestrado). Pontifícia Universidade Católica do Rio Grande do Sul, Porto Alegre, RS.

Kopittke, A. L. W. (2019). Cinco Princípios sobre o que funciona para reduzir a violência. In Proceedings of Sociology of Law 2019: o direito na sociedade tecnológica (pp. 31-650). Canoas, RS: Unilasalle.

Kugley, S., Wade, A., Thomas, J., Mahood, Q., Jørgensen, A. M. K., Hammerstrøm, K., \& Sathe, N. (2016). Searching for studies: a guide to information retrieval for Campbell Systematic Reviews. Campbell Systematic Reviews, 13(1), 1-73

Kume, L. (2004). Uma estimativa dos determinantes da taxa de criminalidade brasileira: uma aplicação em painel dinâmico. Rio de Janeiro, RJ: FGV EPGE.

Lei $n$. 10.826, de 22 de dezembro de 2003. (2003). Dispõe sobre registro, posse e comercialização de armas de fogo e munição, sobre o Sistema Nacional de Armas - Sinarm, define crimes e dá outras providências. Brasília, DF.

Lei n. 11.340, de 7 de agosto de 2006. (2006). Cria mecanismos para coibir a violência doméstica e familiar contra a mulher, nos termos do $₫ 8^{\circ}$ do art. 226 da Constituição Federal, da Convenção sobre a Eliminação de Todas as Formas de Discriminação contra as Mulheres e da Convenção Interamericana para Prevenir, Punir e Erradicar a Violência contra a Mulher; dispõe sobre a criação dos Juizados de Violência Doméstica e Familiar contra a Mulher; altera o Código de Processo Penal, o Código Penal e a Lei de Execução Penal; e dá outras providências. Brasília, DF.

Lipsey, M. W. (2009). The primary factors that characterize effective interventions with juvenile offenders: a meta-analytic overview. Victims and Offenders, 4(2), 124-147.

Lipsey, M. W., Landenberger, N. A., \& Wilson, S. J. (2007). Effects of cognitive-behavioral programs for criminal offenders. Campbell Systematic Reviews, 6, 1-27.

Loschi, R. H., Gonçalves, F. B., \& Cruz, F. R. B. (2005). Avaliação de uma medida de evidência de um ponto de mudança e sua utilização na identificação de mudanças na taxa de criminalidade em Belo Horizonte. Pesqui. Oper., 25(3), 449-463.
Loureiro, A. (2012). Can Conditional Cash Transfers Reduce Poverty and Crime? Evidence from Brazil. Edinburgh, UK: University of Edinburgh.

Loureiro, A. O. F., \& Carvalho, J. R. (2006). O impacto dos gastos públicos sobre a criminalidade no Brasil (Ensaios sobre Pobreza do CAEN, 9). Fortaleza, CE: CAEN.

Lugarinho, L. P., Avanci, J. Q., \& Pinto, L. W. (2017). Perspectivas dos estudos sobre violência na adolescência e cortisol: revisão bibliográfica sistemática. Ciência \& Saúde Coletiva, 22, 1321-1332.

Lum, C., \& Koper, C. S. (2017). Evidence-based policing: translating research into practice. Oxford, England: Oxford University Press.

Lum, C., Koper, C. S., \& Telep, C. W. (2011). The evidence-based policing matrix. Journal of Experimental Criminology, 7(1), 3-26.

Madaleno, M., \& Waights, S. (2015). Guide to scoring methods using the Maryland scientific methods scale. London, England: What Works Centre for Local Economic Growth.

Magaloni, B., Franco, E., \& Melo, V. (2015). Killing in the slums: an impact evaluation of police reform in Rio de Janeiro (Working Paper No. 556). Palo Alto, CA: Stanford.

Marasca, A. R., Colossi, P. M., \& Falcke, D. (2013). Violência conjugal e família de origem: uma revisão sistemática da literatura de 2006 a 2011. Temas em Psicologia, 21(1), 221-243.

Marino, J. M. F. (2012). Fundamentos do 'Paradigma Metodológico Causal' nas Ciências Sociais, Impropriamente denominado 'Métodos Quantitativos'. Sociologias, 14(31), 20-50.

Marvell, T. B., \& Moody, C. E. (1996). Specification problems, police levels, and crime rates. Criminology, 34(4), 609-646.

Matta, R. A., Andrade, M. V. (2005). Economic evaluation of the impact of program control homicide Stay Alive. In Proceedings of the 33rd Brazilian Economics Meeting, Niterói, RJ.

Minayo, M. C. D. S., Souza, E. R. D., \& Paula, D. D. R. D. (2010). Revisão sistemática da produção acadêmica brasileira sobre causas externas e violências contra a pessoa idosa. Ciência \& Saúde Coletiva, 15, 2719-2728. 
Ministério da Defesa. (2018). Pedido de Acesso à Informação 60502000911201845. Brasília, DF: Autor.

Moher D., Liberati A., Tetzlaff J., Altman D. G. (2009) Preferred Reporting Items for Systematic Reviews and Meta-Analyses: The PRISMA Statement. PLoS Med, 6(7): e1000097.

Muggah, R., \& Aguirre, K. (2013). Mapping citizen security interventions in Latin America: reviewing the evidence (NOREF Report). Oslo, Norway: Norwegian Peacebuilding Resource Centre.

Murray, J., Castro Cerqueira, D. R., \& Kahn, T. (2013). Crime and violence in Brazil: systematic review of time trends, prevalence rates and risk factors. Aggression and Violent Behavior, 18(5), 471-483.

Nessello, F., Sant'Anna, F. L., dos Santos, H. G., de Andrade, S. M., Mesas, A. E., \& González, A. D. (2014). Características da violência escolar no Brasil: revisão sistemática de estudos quantitativos. Revista Brasileira de Saúde Materno Infantil, 14(2).

Newman, O., \& Franck, K. A. (1982). The effects of building size on personal crime and fear of crime. Population and Environment, 5(4), 203-220.

Oliveira, C., \& César Rostirolla, C. (2017). Mais armas de fogo, mais homicídios? Uma evidência empírica para a Região Metropolitana de Porto Alegre a partir de dados em painel. In Anais do 45o Encontro Nacional de Economia, Natal, RN.

Oliveira, J. C. L. (2016). Avaliação dos resultados do Pacto pela Vida e a dinâmica dos homicídios nos municípios de Pernambuco (Dissertação de mestrado). Universidade Federal de Pernambuco, Recife, PE.

Organização das Nações Unidas para a Educação, a Ciência e a Cultura. (2005). Vidas Poupadas: o impacto do desarmamento no Brasil. Paris, France: Autor. Recuperado de http://unesdoc.unesco.org/ images/0014/001408/140846por.pdf\%0A

Ottoni, B., \& Ferraz, C. (2014). Três ensaios em microeconometria sobre crime, política e migração (Tese de Doutorado). Pontifícia Universidade Católica do Rio de Janeiro. Rio de Janeiro, RJ.

Patias, N. D., Bossi, T. J., \& Dell'Aglio, D. D. (2014). Repercussões da exposição à violência conjugal nas características emocionais dos filhos: revisão sistemática da literatura. Temas em Psicologia, 22(4), 901-915.

Peixoto, B. T., Andrade, M. V., \& Azevedo, J. P. (2008a). Avaliação econômica do Programa Fica Vivo: o caso piloto (Texto para Discussão Cedeplar n. 336). Belo Horizonte, MG: Universidade Federal de Minas Gerais.

Peixoto, B. T., Andrade, M. V., \& Azevedo, J. P. (2008b). Prevenção e controle de homicídios: uma avaliação de impacto no Brasil. Belo Horizonte, MG: Universidade Federal de Minas Gerais.

Penha, E. F. F., Pontes, E., Jr., \& Vasconcelos, A. L. F. S. (2013). Políticas de Segurança Pública em Combate à Criminalidade no Estado de Pernambuco, Brasil. Recuperado de http://docplayer.com.br/14598334Politicas-deseguranca-publica-em-combate-acriminalidade-no-estado-de-pernambuco-brasil.html.

Pereira, O. A., Filho. (2016). Três ensaios sobre mensuração de eficiência e avaliação de impacto em serviços de segurança pública no Brasil (Tese de Doutorado). Universidade de Brasília, Brasília, DF.

Perova, E., \& Reynolds, S. (2015). Women's Police Stations and Domestic Violence. Policy Research (Working Paper 7497). Washington, DC: World Bank Group.

Ramos, M. P. (2013). Métodos quantitativos e pesquisa em ciência social: lógica e utilidade do uso da quantificação nas explicações dos fenômenos sociais. Mediações - Revista de Ciências Sociais, 18(1), 55-65.

Ramos, M. P. (2014). Pesquisa social: abordagem quantitativa com uso do SPSS. Porto Alegre, RS: Escritos.

Ramos, M. P., \& Schabbach, L. M. (2012). O Estado da arte da avaliação de políticas públicas: conceituação e exemplos no Brasil. Revista de Administração Pública, 46(5), 1271-1294.

Ratton, J. L., \& Daudelin, J. (2018). Construction and Deconstruction of a Homicide Reduction Policy: The Case of Pact for Life in Pernambuco, Brazil. International Journal of Criminology and Sociology, 7, 173-183.

Ribeiro, L., \& Teixeira, A. N. (2017) O calcanhar de Aquiles dos estudos sobre crime, violência e dinâmica criminal. Revista Brasileira de Informação Bibliográfica em Ciências Sociais - BIB, 84(2), 13-80. 
Rolim, M. (2006). A sindrome da rainha vermelha. Policiamento e segurança pública no século XXI. Rio de Janeiro, RJ: Jorge Zahar.

Sá, S. D., \& Werlang, B. S. G. (2013). Personalidade de mulheres vítimas de violência doméstica: uma revisão sistemática da literatura. Contextos Clínicos, 6(2), 106-116.

Sachsida, A., \& Mendonça, M. (2013). Evolução e Determinantes da Taxa de Homicidios no Brasil (Texto para discussão 1808). Rio de Janeiro, RJ: Instituto de Pesquisa Econômica Aplicada.

Sachsida, A., \& Mendonça, M. (2014). Combatendo Homicídios no Brasil: O que Funciona em São Paulo Funciona na Bahia? (Discussion Papers, 1979). Rio de Janeiro, RJ: Instituto de Pesquisa Econômica Aplicada.

Santos, M. J. (2009). Dinâmica temporal da criminalidade: mais evidências sobre o efeito inércia nas taxas de crimes letais nos estados brasileiros. Revista Economia/ANPEC, 10(1), 170-194.

Santos, T., \& Rosa, M. I. (2017). Levantamento Nacional de Informações Penitenciárias (Infopen). Brasília, DF: Departamento Penitenciário Nacional.

Schein, S., \& Martins, R. V. (2013). Revisão sistemática sobre a atenção à saúde da mulher vítima de violência (Monografia de Especialização Lato Sensu). Universidade Federal de Santa Maria, Santa Maria, RS.

Schiavon, L. C. (2017). Essays on crime and justice (Tese de Doutorado). Pontifícia Universidade Católica do Rio de Janeiro, Rio de Janeiro, RJ.

Schneider, A. A. (2005). Determinantes da criminalidade na Região Metropolitana de São Paulo (Dissertação de Mestrado). Fundação Getulio Vargas, São Paulo, SP.

Schucan-Bird, K., Vigurs, C., Quy, K., \& Gough, D. (2016). Criminal justice interventions with perpetrators or victims of domestic violence: a systematic map of the empirical literature (Crime Reduction Systematic Review Series No 3). London, England: What Works Centre for Local Economic Growth.

Schuch, R. C. (2017). Determinantes da Criminalidade na Região Metropolitana de Porto Alegre-RS: Teorias e Evidências. Dissertação defendida ao Programa de Pós-Graduação em Economia da UFRGS. Porto Alegre, RS.

Scorzafave, L. G., Soares, M. K., \& Dorigan, T. A. (2015). Vale a pena pagar para desarmar? Uma avaliação do impacto da campanha de entrega voluntária de armas sobre as mortes com armas de fogo. Estud. Econ., 45(3), 475-497.

Scripilliti, E. A. O. (2006). Crimes nos municípios paulistas: um estudo acerca dos condicionantes sócioeconômico e demográficos que contribuem para maior criminalidade e quais os efeitos das diferentes políticas (Dissertação de Mestrado). Universidade de São Paulo, São Paulo, SP.

Seguridad, Justicia y Paz. (2019). Las 50 ciudades más violentas del mundo 2018. México, DF: Autor.

Sherman, L. W. (1998). Evidence-based policing. Ideas in American policing. Washington, DC: Police Foundation.

Sherman, L. W., Gottfredson, D. C., MacKenzie, D. L., Eck, J., Reuter, P., \& Bushway, S. (1997). Preventing crime: what works, what doesn't, what's promising: a report to the United States Congress. Washington, DC: US Department of Justice.

Sherman, L. W., \& Weisburd, D. (1995). General deterrent effects of police patrol in crime "hot spots": a randomized, controlled trial. Justice Quarterly, 12(4), 625-648.

Silva, B. F. A., Queiroz, B. L., Marinho, F. C., Caminhas, D. A., Pereira, F. N. A., Cisalpino, P. ... \& Gomes, G. (2016). Políticas públicas de prevenção e redução de homicidios: a experiência do "Fica Vivo", em Belo Horizonte e "Pacto pela Vida", em Pernambuco (Relatório Final do Projeto Homicídios no Brasil). Belo Horizonte, MG: Ministério da Justiça; Secretaria Nacional de Segurança Pública; Programa das Nações Unidas para o Desenvolvimento.

Silva, D. R., Neto, Cerqueira, D. R. C., \& Coelho, D. S. C. (2018). Avaliação de política pública para redução de homicídio: um estudo de caso do Programa Estado Presente do Espírito Santo. In Anais do 460 Encontro Nacional de Economia, Rio de Janeiro, RJ.

Silva, L. E. L. D., \& Oliveira, M. L. C. D. (2015). Violência contra a mulher: revisão sistemática da produção científica nacional no período de 2009 a 2013. Ciência \& Saúde Coletiva, 20, 3523-3532. 
Silveira, A M. (2008). A Prevenção de Homicídios: a experiência do Programa Fica Vivo no Morro das Pedras. Revista Educação \& Realidade, 33(2), 163-176.

Silveira, A. M., Assunção, R. M., Silva, B. A. F., \& Beato, C. C., Filho. (2010). Impacto do Programa Fica Vivo na redução dos homicídios em comunidade de Belo Horizonte. Rev. Saúde Pública, 44(3), 496-502.

Silveira, R. M., Filho, Ratton, J. L., Menezes, T. A., \& Monteiro, C. (2014). Avaliação De Política Pública Para Redução Da Violência: O Caso Do Programa Pacto Pela Vida Do Estado De Pernambuco. In Anais do 41 Encontro Nacional de Economia, Brasília, DF.

Soares, L. E. (2006). Legalidade libertária. Rio de Janeiro, RJ: Lumen Juris.

Soares, R., \& Viveiros, I. (2010). Organization and information in the fight against crime: an evaluation of the integration of police forces in the State of Minas Gerais, Brazil (Discussion Paper Series, No. 5270). Bonn, Germany: Institute for the Study of Labor.

Souza, M. F. M., Macinko, J., Alencar, A. P., Malta, D. C., \& Morais, O. L. M., Neto. (2007). Reductions In Firearm-Related Mortality And Hospitalizations In Brazil After Gun Control. Health Affairs, 26(2), 575-584.

Souza, T. A. (2016). A implementação da política estadual de prevenção social à criminalidade de Minas Gerais: paradigmas, coalizões e incrementalismo (Dissertação de Mestrado). Fundação João Pinheiro, Belo Horizonte, MG.

Spelman, W., \& Brown, D. K. (1981). Calling the police: citizen reporting of serious crime. Washington, DC: Police Executive Research Forum.

Telep, C. W. (2009). Citation Analysis of Randomized Experiments in Criminology and Criminal Justice: A research note. Journal of Experimental Criminology, $5,441-64$.

Vasconcellos, L., \& Menezes, N., Filho. (2005). Relatório de Avaliação Econômica - Programa Escola da Família da Secretaria de Educação do Estado de São Paulo. São Paulo, SP: Fundação Itaú Social.
Volpe, F. M., Ladeira, R. M., \& Fantoni, R. (2017). Evaluating the Brazilian zero tolerance drinking and driving law: Time series analyses of trafficrelated mortality in three major cities. Traffic Injury Prevention, 18(4), 337-343.

Waar, J. (2019, julho). What Works? A systematic overview of recently published meta evaluations / synthesis studies within the knowledge domains of Situational Crime, Prevention, Policing and Criminal Justice Interventions, 1997-2019 (Working Paper).

Waiselfisz, J. J. (2015). Mapa da violência 2015: adolescentes de 16 e 17 anos do Brasil: mortes matadas por armas de fogo. Brasília, DF: Centro Brasileiro de Estudos Latino-Americano. Recuperado de http://www.mapadaviolencia.org.br/pdf2015/ mapaViolencia2015.pdf\%0A

Weikart, D. P., Deloria, D., Lawser, S., \& Wiegerink, R. (1970). Longitudinal results of the Ypsilanti Perry Preschool Project (Monographs of the High/Scope Educational Research Foundation, No. 1). Ypsilanti, MI: High/Scope Press.

Weisburd, D., Farrington, D. P., \& Gill, C. (2017). What works in crime prevention and rehabilitation: an assessment of systematic reviews. Criminology \& Public Policy, 16(2), 415-449.

Weisburd, D., Lum, C. M., \& Petrosino, A. (2001). Does research design affect study outcomes in criminal justice? The Annals of the American Academy of Political and Social Science, 578(1), 50-70.

Welsh, Brandon C., \& Farrington, David P. (2012). Crime Prevention and Public Policy. The Oxford Handbook of Crime Prevention. Oxford, UK: Oxford Handbooks.

Willadino, R., Sento-Sé, J. T., Dias, C. G., \& Gomes, F. (2011). Prevenção à violência e redução de homicídios de adolescentes e jovens no Brasil. Rio de Janeiro, RJ: Observatório de Favelas.

Zaverucha, J., \& Nóbrega, J. M. P., Jr. O Pacto pela Vida, os tomadores de decisão e a redução da violência homicida em Pernambuco. Dilemas: Revista de Estudos de Conflito e Controle Social, 8(2), 235-252. 


\section{Alberto L. W. Kopittke}

https://orcid.org/0000-0003-1264-0956

Graduação em Direito pelo Centro Universitário do Distrito Federal (UDF); Mestrado em Ciências Criminais pela Pontifícia Universidade Católica do Rio Grande do Sul (PUCRS); Doutorado em Políticas Públicas pela Universidade Federal do Rio Grande do Sul (UFRGS); Consultor do Banco Interamericano de Desenvolvimento (BID) e membro do Instituto Cidade Segura. E-mail: albertopoa2012@gmail.com

\section{Marília Patta Ramos}

https://orcid.org/0000-0003-0880-8269

Licenciatura e Bacharelato em Ciências Sociais pela Universidade Federal do Rio Grande do Sul (UFRGS); Mestrado em Sociologia pela Universidade Federal do Rio Grande do Sul (UFRGS); Doutoramento em Sociologia pela Purdue University; Pós-doutoramento pelo Population Research Center (PRC) da Universidade do Texas; Professora Associada no Departamento de Sociologia da Universidade Federal do Rio Grande do Sul (UFRGS). E-mail: mariliaramos68@gmail.com 